\title{
Biomarkers for gastrointestinal immunotherapy-related adverse events
}

\author{
Shek $D^{*}$ \\ Clinical School and Research Centre, Western Sydney University, Blacktown, NSW, 2148, Australia
}

\section{Introduction}

Immune-checkpoint inhibition is proposed as a new promising therapy against cancer. Nowadays there are two types of immunotherapeutic drugs: CTLA-4 (cytotoxic T-lymphocyte antigen-4) and PD-1 (programmed cell death-1) blocking antibodies. The efficacy of anti-CTLA-4 (ipilimumab) and anti-PD-1 (nivolumab and pembrolizumab) has been proven on malignant lesions, such as melanoma, renal cell carcinoma (RCC) and non-small cell lung cancer (NSCLC) [1-3]. The mechanism of action includes the activation of T-cell immune response, which controls cell recognition and tumor's regression. After 3 years of ipilimumab treatment, patient survival rate is almost $21 \%$ [4]. Despite its positive therapeutic effect, such therapy causes a lot of immune-related adverse events (irAEs). Gastrointestinal (GI) complications occur in $12 \%$ of patients, treated by ipilimumab [5]. Patients, who receive anti-PD-1 treatment show less frequency of irAEs. GI complications, induced by immunotherapy, are ranged from diarrhea and inflammatory bowel disease (IBD) to bowel's perforation [6]. In this minireview article we described the existing data for different potential biological and clinical markers of GI immune-related complications which can be used as possible tools for creation effective prophylaxis of GI irAEs in patient on immunotherapeutic drugs.

\section{Possible biomarkers of GI irAEs}

In 2013, group of scientists from the Bristol-Myers Squibb have analyzed 162 melanoma patients, treated by ipilimumab. Only 49 patients had GI complications grade II or higher. It was discovered, that GI complications mostly start to occur after second or third dose of ipilimumab's administration. Their study also revealed the fact, that neutrophil surface protein CD 177 (cluster of differentiation), which is important in neutrophil's activation, was highly increased on the third week of treatment, comparing to baseline levels on the 0 week and to the patients without GI irAEs. It was proposed, that CD 177 could play a role of early predictor of gastrointestinal immunotherapeutic complications. It was also reported, that on the 11th week some immunoglobulin-related genes, such as IGHA1, IGHA2, IGHG1, IGHV4-31 notably activated in the ipilimumab-treated patients with GI complications, comparing to their baseline levels in the beginning of the treatment.

CEACAM1 (carcinoembryonic antigen-related cell adhesion molecule 1) is another prospective predictive marker of GI complications. It started to increase after three weeks, from the first ipilimumab's administration especially in patients with GI adverse events [7].

It worth mentioning, that female sex has higher risk of immunerelated adverse events' development. As demonstrated by Valpione.S. and co-authors, among 140 ipilimumab-treated patients, 26\% had severe adverse events and $24 \%$ left the treatment due to the toxicity. Most of the patients with gastrointestinal adverse events were female with low baseline level of interleukin-6, which is associated with immune response and produced by activated T-cells or macrophages [8].

In addition to interleukin-6, another cytokine Il-17 has been identified as possible marker for gastrointestinal irAEs in ipilimumabtreated patients. It was found, that among patients with grade III diarrhea or colitis, IL-17 was highly increased, comparing to its baseline level $(p=0,02)$. These results were measured between 0 and 6 th weeks [9]

Autoantibodies against CTLA- 4 could also play role as a biomarker of treatment response. It was shown, that among 37 patients with metastatic melanoma, treated by ipilimumab, 28 had different irAEs with increased level of IgG autoantibodies [10].

Anti-PD-1 drugs, such as nivolumab and pembrolizumab, are considered to be more safe and effective immunotherapeutic drugs, which better prolongs survival of patients with metastatic melanoma. Despite that, the pilot study revealed [11], that among 46 patients, treated by nivolumab, $45,7 \%$ had different complications. Before the first administration of the drug, serum level of CD163 (cluster of differentiation) and CXCL5 (C-X-C motif chemokine ligand 5) was measured. On the $42 \mathrm{nd}$ day of treatment serum level of both markers was increased, but CD163 was highly increased in patients with adverse events [11].

These results are very promising and require further clinical validation on larger group of patients.

\section{Gut microbiota: biomarker or not?}

Does gut flora play any role in gastrointestinal immune-related complications? In 2015, the experiment provided on mice has shown that tumors become resistant to the anti-CTLA- 4 treatment in case of unhealthy gut flora [12]. The immunogenic bacteria Bacteroides fragilis was proposed as predictor of effective treatment by ipilimumab [13]. The authors suggested that the higher concentration of the $\mathrm{B}$. fragilis could lead to the better treatment response. From the other side, this data suggests, that disruption in ratio of gut flora, by increase of B. fragilis, could lead to dysbiosis, which, in turn, could result in colitis or inflammatory bowel disease and be considered as a GI complication during immunotherapy. However, this point of view requires further study.

${ }^{*}$ Correspondence to: Dmitrii Shek, Clinical School and Research Centre, Western Sydney University, Blacktown, NSW, 2148, Australia, E-mail 19238627@student.westernsydney.edu.au

Received: July 18, 2018; Accepted: July 23, 2018; Published: August 09, 2018 


\section{Discussion}

Several studies have shown that there is a correlation between immune-related adverse events and response to therapy. Judd J. and Zibelman M. have demonstrated, that patients with low-grade complications after anti-PD-1 immunotherapeutic treatment, have better response to the treatment $(p=0,017)$ and longer TTNTD (time to next therapy or death, $\mathrm{p}=0,008)$. However, the correlation between presence of irAEs, OS (overall survival) and mortality rate in groups of patients with or without immune-related complications haven't been described [14]. Another research project had a pool of 148 patients with melanoma treated by nivolumab. $68,2 \%$ of patients had irAEs of different severity. The study has shown, that skin irAEs, such as rash and vitiligo, correlated with better OS of patients with metastatic melanoma $(p=0,004)$. Nevertheless, the correlation of other irAEs, such as gastrointestinal, with OS of patients haven't been studied [15]. Even though irAEs are associated with better response rate and skin irAEs with higher overall survival, the patient's quality of life, receiving immune checkpoint inhibitors should be also considered. Diarrhea, colitis and other gastrointestinal immune-related adverse events could not just worsen patient's quality of life, but also lead to other complications, which, in turn, could lead to sudden non-oncological death. Hence, identifying predictive biomarkers for immune-related adverse events is very vital for modern immunoncology. The prevention program for the most common adverse gastrointestinal events based on specific biomarkers will definitely benefit existing protocols for cancer immunotherapy.

\section{Conclusion}

Thus, current data presents different spectrum of possible biological and clinical markers correlated with the gastrointestinal irAEs. Biomarkers CD177 and CD163 should be considered as the most trustworthy markers among all, according to the existing information. However, gastrointestinal irAEs should produce numerous detectable genetic and epigenetic alterations. Further research, which will include large cohort of patients receiving different immune checkpoint inhibitors in different dosages and new bio-medical tools should be conceded. Without doubt, the discovery of specific and unique biomarkers for gastrointestinal immune-related adverse events will help to create effective methods for prophylaxis of irAEs and, as a result, immunotherapy will become not just effective, but also safe and easy tolerable treatment for oncological patients.

\section{Acknowledgement}

I would like to thank Prof Golo Ahlenstiel and Dr Irina Piatkov for their support in article preparation.

\section{References}

1. Ascierto PA, Brugarolas J, Buonaguro L, Butterfield LH, Carbone D, et al. (2018) Perspectives in immunotherapy: meeting report from the immunotherapy bridge (2930 November, 2017, Naples, Italy). J Immunother Cancer 6: 69. [Crossref]

2. Beavis PA, Henderson MA, Giuffrida L, Davenport AJ, Petley EV, et al. (2018) Dual PD-1 and CTLA-4 checkpoint blockade promotes antitumor immune responses through CD4+Foxp3- cell-mediated modulation of CD103+ dendritic cells. Cancer Immunol Res. [Crossref]

3. Hargadon KM, Johnson CE, Williams CJ (2018) Immune checkpoint blockade therapy for cancer: An overview of FDA-approved immune checkpoint inhibitors. Int Immunopharmacol 62: 29-39. [Crossref]

4. Schadendorf D, Hodi FS, Robert C, Weber JS, Margolin K, et al. (2015) Pooled analysis of long-term survival data from phase II and phase III trials of Ipilimumab in unresectable or metastatic melanoma. J Clin Oncol 33: 1889-1894. [Crossref]

5. Hodi FS, O'Day SJ, McDermott DF, Weber RW, Sosman JA, et al. (2010) Improved survival with ipilimumab in patients with metastatic melanoma. $N$ Engl $J$ Med 363: 711-723. [Crossref]

6. Wang ZH, Shen L (2018) Management of gastrointestinal adverse events induced by immune-checkpoint inhibitors. Chronic Dis Transl Med 4: 1-7. [Crossref]

7. Shahabi V, Berman D, Chasalow SD, Wang L, Tsuchihashi Z, et al. (2013) Gene expression profiling of whole blood in ipilimumab-treated patients for identification of potential biomarkers of immune-related gastrointestinal adverse events. $J$ Transl Med 11: 75. [Crossref]

8. Valpione S, Pasquali S (2018) Sex and interleukin-6 are prognostic factors for autoimmune toxicity following treatment with anti-CTLA4 blockade. J Transl Med 16: 94. [Crossref]

9. Tarhini AA, Zahoor H, Lin Y, Malhotra U, Sander C, et al. (2015) Baseline circulating IL-17 predicts toxicity while TGF-I $\hat{I}^{2} 1$ and IL-10 are prognostic of relapse in ipilimumab neoadjuvant therapy of melanoma. J Immunother Cancer 3: 39. [Crossref]

10. Patil PD, Burotto M, Velcheti V (2018) Biomarkers for immune-related toxicities of checkpoint inhibitors: current progress and the road ahead. Expert Rev Mol Diagn 18: 297-305. [Crossref]

11. Fujimura T, Sato Y, Tanita K, Kambayashi Y, Otsuka A, et al. (2018) Serum levels of soluble CD163 and CXCL5 may be predictive markers for immune-related adverse events in patients with advanced melanoma treated with nivolumab: a pilot study. Oncotarget 9: 15542-15551. [Crossref]

12. Vétizou M, Pitt JM, Daillère R, Lepage P, Waldschmitt N, et al. (2015) Anticancer immunotherapy by CTLA-4 blockade relies on the gut microbiota. Science 350: 10791084. [Crossref]

13. Cramer P, Bresalier RS (2017) Gastrointestinal and hepatic complications of immune checkpoint inhibitors. Curr Gastroenterol Rep 19: 3. [Crossref]

14. Judd J, Zibelman M, Handorf E, O’Neill J, Ramamurthy C, et al. (2017) Immune-related adverse events as a biomarker in non-melanoma patients treated with programmed cell death 1 inhibitors. Oncologist 22: 1232-1237. [Crossref]

15. Freeman-Keller M, Kim Y, Cronin H, Richards A, Gibney G, et al. (2016) Nivolumab in resected and unresectable metastatic melanoma: Characteristics of immune-related adverse events and association with outcomes. Clin Cancer Res 22: 886-894.

Copyright: (C2018 Shek D. This is an open-access article distributed under the terms of the Creative Commons Attribution License, which permits unrestricted use, distribution, and reproduction in any medium, provided the original author and source are credited. 